\title{
RUDJER BOŠKOVIĆ INSTITUTE RADIOCARBON MEASUREMENTS III
}

\section{DUŠAN SRDOČ, ADELA SLIEPČEVIC,* and JOSIP PLANINIC***}

Rudjer Bošković Institute, P O B 1016, 41001 Zagreb, Yugoslavia

The following list contains dates of samples measured since our previous list ( $\mathrm{R}, 1973$, v 15, p 435-441). As before, age calculations are based on the Libby half-life $5568 \pm 30 \mathrm{yr}$, and reported in years before 1950. The modern standard is 0.950 of the activity of NBS oxalic acid.

Before combustion, wood and charcoal were treated with $4 \% \mathrm{HCl}$. Samples of soil with high percentage of limestone were treated with $50 \%$ HCl. Samples containing recent carbon (mold, rootlets) were boiled in $4 \% \mathrm{NaOH}$. The counting method is essentially the same as described in $\mathrm{R}, 1971, \mathrm{v} 13, \mathrm{p}$ 135-140. Sample descriptions were prepared with collectors and submitters. The errors quoted correspond to $1 \sigma$ variation of sample net counting rate and do not include the uncertainty in ${ }^{14} \mathrm{C}$ half-life. Data are not corrected for isotopic fractionation. The recent activity of speleothems (dripstones) is assumed to be $85 \%$ of modern samples; therefore $1305 \mathrm{yr}$ was subtracted from the radiocarbon age (Münnich and Vogel, 1959).

\section{ACKNOWLEDGMENTS}

We thank E Hernaus for preparation of samples and methane synthesis, B Obelić, and B Novak for data processing, and V Andreić for construction and maintenance of gas lines and electronics.

\section{Selevac series}

\section{ARCHAEOLOGIC SAMPLES}

Charred grain (Triticum sp) from a baked earth receptacle that rested on lowest occupation floor, underlying several occupation levels and overlying ashy layer devoid of artifacts, in Sonda S-VII at Selevac, loc "Staro Selo", near Smederevska Palanka $\left(44^{\circ} 30^{\prime} \mathrm{N}, 20^{\circ} 53^{\prime} \mathrm{E}\right)$, N Serbia. Site spans most of Serbian Middle and Late Neolithic sequence. Figurine found in receptacle, the only artifact, is diagnostic of Vinča "A" at type site. Coll 1970 by Radovan Milošević and Vojislav Novaković, Nat Mus Smederevska Palanka, and subm by A McPherron, Univ Pittsburgh.

\section{Z-233A. Selevac}

$6113 \pm 80$

Whole unbroken grains treated with $4 \% \mathrm{HCl}$ and $4 \% \mathrm{NaOH}$.

\section{Z-233B. Selevac}

$6152 \pm 90$

Whole unbroken grains without chemical treatment; mean value of 2 measurements.

* Faculty of Veterinary Medicine, Univ Zagreb, Yugoslavia

** Faculty of Pharmacy and Biochemistry, Univ Zagreb, Yugoslavia 


\section{Z-233. Selevac}

Rest of sample, treated with $4 \% \mathrm{HCl}$ and $4 \% \mathrm{NaOH}$. Comments (DS): while both samples gave very close dates, whole-grains samples are regarded as more reliable; (AM): date confirms early position of occupation level in Vinča sequence.

\section{Z-234. Varaždinske toplice}

$283 \pm 60$

\section{AD 1667}

Fragments of wood from draw-well $2 \mathrm{~m}$ below surface $\left(46^{\circ} 14^{\prime} \mathrm{N}\right.$, $14^{\circ} 05^{\prime}$ E). Sample coll 1973 by Marcel Gorenc, Archaeol Mus, Zagreb.

\section{Z-269. Varaždinske toplice}

$1533 \pm 75$

\section{AD 417}

Fragments of charred wood, remains of rafter from NW capitol of

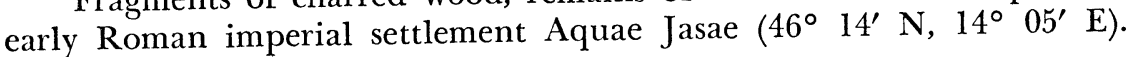
Coll 1973 and subm by Marcel Gorenc.

\section{Z-236. Hutovo Blato}

\section{AD 1520}

$$
430 \pm 60
$$

Fragment of wooden boat (Quercus) $11 \mathrm{~m}$ below surface, spring Desilo, Hutovo Blato $\left(43^{\circ} 3^{\prime} \mathrm{N}, 17^{\circ} 45^{\prime} \mathrm{E}\right)$ near Čapljina, Hercegovina. Coll 1972 and subm by Vukosava Atanacković-Salčić, Inst Protection Cultural Monuments, Regional Center, Mostar.

\section{Hrvatska Dubica series}

Fragments of wooden boat from muddy bed of Una R near Hrvatska Dubica $\left(45^{\circ} 11^{\prime} \mathrm{N}, 16^{\circ} 49^{\prime} \mathrm{E}\right)$ Croatia. Coll 1973 and subm by Members Regional Inst Protection Cultural Monuments, Zagreb.

\section{Z-251. Hrvatska Dubica I}

\section{Z-255. Hrvatska Dubica II}

\section{Z-256. Bosanska Gradiška}

Fragment of wooden boat from sandy bed of Jablanica R NW of Bosanska Gradiška (45 $\left.7^{\prime} 30^{\prime \prime} \mathrm{N}, 17^{\circ} 11^{\prime} 30^{\prime \prime} \mathrm{E}\right)$. Coll 1973 and subm by Drago Malešević, Bosanska Gradiška.

\section{Z-257. Dubrovnik, Knežev dvor}

Wooden pillar from foundation of Duke's Palace (Knežev Dvor). Found during restoration of palace. Date important for chronology of building.

\section{Z-260. Lopar}

AD 1756

$$
194 \pm 60
$$

Charcoal from hearth, Lopar $\left(44^{\circ} 51^{\prime} \mathrm{N}, 14^{\circ} 43^{\prime} \mathrm{E}\right)$ on Rab I. Coll 1972 and subm by Vjeko Legac, Rab. 
Charcoal from layer in Illyrian iron melting-furnace in Blagaj near Bosanski Novi $\left(45^{\circ} 0^{\prime} \mathrm{N}, 16^{\circ} 25^{\prime} \mathrm{E}\right)$. Depth: $2 \mathrm{~m}$ below dross layer in gray clay. Coll 1973 and subm by Djuro Basler, Regional Mus, Sarajevo. Comment $(\mathrm{Dj} \mathrm{B})$ : expected age: $70 \mathrm{BC}$ to $\mathrm{AD} 50$.

\section{Vlasac series}

Charcoal from archaeol excavation of Mesolithic settlement Vlasac $\left(44^{\circ} 32^{\prime} \mathrm{N}, 22^{\circ} 3^{\prime} \mathrm{E}\right)$ near Donji Milanovac. Settlement discovered during construction of Djerdap hydro-electric power plant. Coll 1970 and subm by Dragoslav Srejović and Zagorka Letica, Fac Arts \& Sci, Archaeol Dept, Belgrade.

\section{Z-262. Vlasac I}

$7000 \pm 90$

Charcoal from hearth, House I, Sonda A, Level XXVI, depth $4.1 \mathrm{~m}$ below surface, oldest horizon. Comment (DS): dates agree with expected period (Mesolithic, 7-6th millennium BC).

\section{Z-264. Vlasac I}

$6335 \pm 92$

Charcoal from Burial 54, Quad A/17, Level XI.

\section{BC}

\section{Z-267. Vlasac II}

$7559 \pm 93$

5609 BC

Charcoal below Hearth 16. Comment (DS): expected age: end of Mesolithic, 7-6th millennium Bc.

Z-268. Vlasac ?

Charcoal from Burial 11, Quad a/6, Level VII.

\section{Z-270. Vela Svitnja}

Fragments of charred wood (Pinus) from sand at depth $35 \mathrm{~m}$, Vela Svitnja, Vis bay $\left(43^{\circ} 04^{\prime} \mathrm{N}, 16^{\circ} 12^{\prime} \mathrm{E}\right)$, Vis I. Coll 1973 and subm by Nenad Cambi, Archaeol Mus, Split.

\section{Z-283. Ščitarjevo}

$1949 \pm 77$

AD 1

Charcoal from cult fireplace in burial place, Block 7 , depth $1 \mathrm{~m}$, in Roman municipality Andautonia near Zagreb (45 $\left.46^{\prime} \mathrm{N}, 16^{\circ} 00^{\prime} \mathrm{E}\right)$. Coll 1973 and subm by Branka Vikić and Marcel Gorenc, Archaeol Mus, Zagreb.

\section{Nin series, Croatia}

Fragments of wooden ship from sea under muddy sand at $2 \mathrm{~m}$ depth at Ždrijac, port of Nin $\left(44^{\circ} 15^{\prime} \mathrm{N}, 15^{\circ} 15^{\prime} \mathrm{E}\right)$. Coll 1973 and subm by Zdenko Brusić and Božidar Vilhar, Archaeol Mus, Zadar. 
$961 \pm 64$

Z-296. Nin I

Hull fragments.

\section{Z-297. Nin II}

Hull fragments of same ship.

AD 989

\section{Z-298. Nin III}

Fragments of wooden rib from same ship.
$937 \pm 71$

AD 1013

$1177 \pm 80$

AD 773

$>35,000$

Z-299. Slavonski Brod

Fragments of wood assoc with steppe elephant (parelephas trogontherii) skull from Glogovica channel near Slavonski Brod $\left(45^{\circ} 10^{\prime} \mathrm{N}\right.$, $18^{\circ} 02^{\prime} 30^{\prime \prime}$ E). Coll 1973 and subm by Mirko Malez, Yugoslav Acad Sci, Zagreb.

\section{Z-306. Hruševje}

$2735 \pm 100$ 785 вC

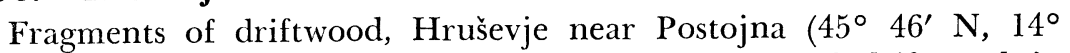
$07^{\prime} 15^{\prime \prime}$ E). Date determines period of accumulation of driftwood in Nanošca creek valley. Coll 1973 and subm by Alojz Šercelj, Slov Acad Sci \& Arts, Ljubljana.

\section{Z-307. Loče}

$7150 \pm 100$

Fragments of driftwood, Loče near Slovenska Bistrica $\left(46^{\circ} 22^{\prime} 45^{\prime \prime}\right.$ $\left.\mathrm{N}, 15^{\circ} 33^{\prime} 30^{\prime \prime} \mathrm{E}\right)$. Date determines period of accumulation of driftwood in Ložnica creek valley. Coll 1973 and subm by Alojz Šercelj.

\section{Ljubljansko Barje series}

Wood fragments from ash (Fraxinus) pilings of pile houses from Ljubljansko Barje peat bog ( $\left.45^{\circ} 58^{\prime} \mathrm{N}, 14^{\circ} 32^{\prime} \mathrm{E}\right)$, Slovenia. Potsherds, estimated age, $3800 \mathrm{yr}$ old, assoc with fragments.

\section{Z-278. Ljubljansko Barje}

$4633 \pm 117$

Z-305. Veliki Mah No. 1

$4345 \pm 113$

\section{Z-314. Maharski prekop}

2395 вс

\section{Z-315. Maharski prekop}

$4964 \pm 99$ 3104 BC

\section{Kaptol series}

Charcoal from rectangular charcoal-filled trench in prehistoric Barrow I in Kaptol village near Požega $\left(45^{\circ} 26^{\prime} \mathrm{N}, 17^{\circ} 44^{\prime} \mathrm{E}\right)$. Results important for chronology of Early Iron age of Požega Valley. Coll 1973 and subm by Vera Vejvoda and Ivan Mirnik, Archaeol Mus, Zagreb. 
Z-316. Kaptol 1

Z-317. Kaptol 2

II. GEOLOGIC SAMPLES

\section{Grapa series, Slovenia}

Calcite from stalagmite, Grapa Cave $\left(46^{\circ} 49^{\prime} \mathrm{N}, 14^{\circ} 09^{\prime} \mathrm{E}\right)$ near Belsko. Coll 1972 and subm by France Habe, Slovenian Acad Sci \& Arts, Postojna. Samples date periods of growth of sinter.

Z-231. Grapa

$6380 \pm 100$

Calcite from stalagmitic core, entrance gallery.

4430 BC

\section{Z-231/I. Grapa}

$3330 \pm 80$

Calcite from same stalagmite, 0 to $4 \mathrm{~mm}$ below surface.

$1380 \mathrm{BC}$

\section{Z-231/II. Grapa}

$4300 \pm 90$

Calcite from same stalagmite, 7 to $12 \mathrm{~mm}$ below surface.

\section{Z-231/IV. Grapa}

$2201 \pm 63$

Calcite from tip of same stalagmite.

$251 \mathrm{BC}$

\section{Z-232/I. Grapa}

$3942 \pm 80$

$1992 \mathrm{BC}$

Calcite from stalagmite grown on clay in $\mathrm{N}$ gallery, 0 to $10 \mathrm{~mm}$ below surface.

\section{Z-232/II. Grapa}

$6252 \pm 100$

Calcite from same stalagmitic core.

\section{Z-227. Grapa}

$2410 \pm 78$

Calcite from stalagmite from $\mathrm{N}$ gallery. Comment $(\mathrm{FH})$ : expected period: Holocene.

\section{Rastuša series}

Calcite from stalactite from top of Rastuša cave near Teslić $\left(44^{\circ}\right.$ $41^{\prime} 48^{\prime \prime} \mathrm{N}, 17^{\circ} 48^{\prime} 20^{\prime \prime} \mathrm{E}$ ). This stratum is sterile, but next layer contains bones of cave bear (Ursus spelaeus). Coll 1972 and subm by Mirko Malez, Yugoslav Acad Sci, Zagreb. Comment (MM): expected period: Pre-Boreal.

\section{Z-238/I. Rastuša}

Calcite from core of stalactite. 


\section{Z-238/II. Rastuša}

Calcite from same stalactite 0 to $2 \mathrm{~cm}$ below surface.

\section{Buško Blato series}

Speleothems from caves discovered during construction of dam, Orlovac hydroelectric plant $\left(43^{\circ} 40^{\prime} \mathrm{N}, 16^{\circ} 59^{\prime} \mathrm{E}\right)$. Dates helped to establish chronology of cave formation, growth of speleothems and tectonic changes. Coll 1972 and subm by Srećko Božičević, Inst Geol, Zagreb.

\section{Z-239/I. Buško Blato 1}

$1857 \pm 74$

Base of submerged stalagmite, Sec 8, of interest for dating formation of sandy layer in stalagmite. Calcite below sand.

\section{Z-239/II. Buško Blato 1}

$1828 \pm 62$

Calcite above sand.

\section{Z-240/I. Buško Blato 2} AD 122

Calcite from core, submerged stalagmite, Sec 8, near siphon.

\section{Z-240/II. Buško Blato 2}

Modern

Calcite from tip of same stalagmite.

\section{Z-241. Buško Blato 3}

Calcite from tip, submerged stalagmite, Sec 8.

\section{Z-243. Buško Blato 5}

Calcite from core of base of broken stalagmite from great hall, Sec 8 , grown on a calcite block.

\section{Z-244/I. Buško Blato 6}

Calcite from stalagmite from Great Hall beneath block with stalagmite Z-243, Sec 8. Calcite from core of base of stalagmite.

\section{Z-244/II. Buško Blato 6}

Calcite from same stalagmite 0 to $5 \mathrm{~cm}$ below surface.

\section{Z-245. Buško Blato 7}

$312 \pm 70$

Calcite from outer layer of stalagmite grown on a block under rock in right canal, Sec 8 .

\section{Z-246/I. Buško Blato 8}

Calcite from stalagmite grown on a block under inclined rock, Sec 17. Calcite from base of stalagmite. 


\section{Z-246/II. Buško Blato 8}

$659 \pm 70$

Calcite from tip of same stalagmite.

AD 1291

Z-247. Buško Blato 11

$3382 \pm 78$

1432 BC

Calcite from base of stalagmite grown on muddy ground behind block with stalagmite Z-246, Sec 17.

\section{Z-248/I. Buško Blato 9}

$2041 \pm 64$

$91 \mathrm{BC}$

Calcite from stalagmite grown on calcite block, Sec 17. Calcite from base of stalagmite.

\section{Z-248/II. Buško Blato 9}

Calcite from tip of same stalagmite.

Calcite from fragment of speleothem $10 \mathrm{~cm}$ thick, Sec 27. Core of speleothem.

\section{Z-249/II. Buško Blato 12}

$20,990 \pm 405$

Outer part of speleothem. Sample mixed with dead gas for counting.

\section{Z-250. Buško Blato 13}

$1945 \pm 94$

Calcite from base of broken stalagmite grown on calcite crust 0 to $6 \mathrm{~mm}$ below surface. Sample mixed with dead gas for counting.

\section{Z-279. Ciganske Jame}

$4790 \pm 120$

2840 вС

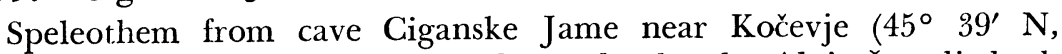
$14^{\circ} 53^{\prime}$ E). Coll 1973 by Mitja Brodar and subm by Alojz Sercelj, both of Slovenian Acad Sci \& Arts, Ljubljana.

\section{REFERENCES}

Münnich, K O and Vogel, J C, 1959, Alterbestimmung von Süsswasser-Kalkablagerungen: Naturwissenschaften, $\mathrm{v} 46, \mathrm{p} 168$.

Srdoč, D, Breyer, B, and Sliepčevic, A, 1971, Rudjer Boškovic Institute radiocarbon measurements I: Radiocarbon, v 13, 1971, p 135-140.

Srdoč, D, Sliepčevic, A, Planinic, J, Obelic, B, and Breyer, B, 1973, Rudjer Boškovic Institute radiocarbon measurements II: Radiocarbon, v 15, 1973, p 435-441. 